Audiol Neurotol 2015;20(suppl 1):73-78

DOI: $10.1159 / 000380752$

\section{Binaural Integration of Periodically Alternating Speech following Cochlear Implantation in Subjects with Profound Sensorineural Unilateral Hearing Loss}

Thomas Wesarga , Nicole Richter ${ }^{\mathrm{a}}$, Horst Hessel ${ }^{\mathrm{b}}$, Stefanie Günther ${ }^{\mathrm{a}}$, Susan Arndt ${ }^{\mathrm{a}}$, Antje Aschendorffa, Roland Lasziga ,

Frederike Hassepass ${ }^{\mathrm{a}}$

a Department of Otorhinolaryngology, Head and Neck Surgery, University Medical Centre Freiburg, Freiburg, and ${ }^{\mathrm{b}}$ Cochlear Deutschland GmbH \& Co. KG, Hannover, Germany

\section{Key Words}

Binaural integration - Cochlear implant - Dichotic stimulation . Speech recognition . Unilateral hearing loss

\begin{abstract}
In cochlear implant $(\mathrm{Cl})$ recipients with unilateral hearing loss (UHL) and normal hearing $(\mathrm{NH})$ in the contralateral ear, the central auditory system receives signals of different auditory modalities, i.e. electrically via the $\mathrm{Cl}$ ear as well as acoustically via the $\mathrm{NH}$ ear. The present study investigates binaural integration of bimodal stimulation in the central auditory system of $10 \mathrm{Cl}$ subjects with UHL by applying a modified version of the Rapidly Alternating Speech Perception (RASP) test to characterise speech recognition ability under monotic and dichotic listening arrangements. Subsequently, the results for each monotic and dichotic test condition were compared to quantify the binaural benefit from $\mathrm{Cl}$ usage. The study results demonstrate significantly improved speech recognition under dichotic compared to monotic listening conditions, providing evidence that there is binaural integration of acoustically and electrically transmitted speech segments in the central nervous system at brainstem and cortical levels. In contrast to more commonly used tests of binaural integration, such as localisation, the RASP test provides the clinical option to investigate binaural integration involving structures at the cortical level.
\end{abstract}

(c) 2015 S. Karger AG, Basel

\section{Introduction}

In cochlear implant (CI)-treated patients with acquired severeto-profound unilateral hearing loss (UHL) and normal or nearnormal hearing $(\mathrm{NH})$ in the contralateral ear, the central auditory system receives signals from the electrically stimulated CI ear as well as from the acoustically stimulated contralateral ear, i.e. signals of different auditory modalities. Previous studies have found a binaural benefit of CI usage in these patients with an improvement in (i) speech recognition in noise [Vermeire and Van de Heyning, 2009; Buechner et al., 2010; Arndt et al., 2011] and (ii) sound localisation performance [Arndt et al., 2011; Jacob et al., 2011]. From the localisation findings as well as from tests of binaural summation [Laszig et al., 2014], it can be concluded that there is binaural processing and representation of acoustically and electrically transmitted stimulus information, particularly interaural level differences in the auditory brainstem in case of diotic stimulus presentation. However, to the authors' knowledge, there is currently no adequate method to test binaural benefit from CI usage in unilaterally deafened CI recipients that involves binaural processing at the cortical level of the central auditory cortex.

The present study in unilaterally deafened CI users addresses the question of whether binaural integration of acoustic and electrical stimulation in the central auditory system, including processing mechanisms assigned to higher-ordered structures that are beyond the auditory brainstem, is associated with dichotic stimulus presentation, i.e. an integration of different partial information presented to each ear to binaural perception of the whole stimulus. Different test paradigms have been used to assess binaural integration based on cognitive processes and neurophysiological correlates at the cortical level [Medwetsky, 2002; Stach, 2007]. One adequate method is the Rapidly Alternating Speech Perception (RASP) test introduced by Bocca and Calearo [1963], wherein speech is segmented and acoustically presented speech segments are alternated periodically between both ears at a certain rate, e.g. 3.33 segments per second (sps), i.e. every $300 \mathrm{~ms}$. Speech recognition is measured for monotic stimulation of either ear and for dichotic stimulation, and the binaural integration can be assessed as the improvement in speech recognition comparing results for dichotic stimulation with results for either monotic stimulation.

Here we applied an enhanced RASP test including specific low, medium, and high segmentation rates to assess monotic and dichotic speech recognition in order to draw conclusions about the ability of binaural integration, which is based on intact processing mechanisms assigned to the brainstem and also to higher-ordered cortical structures in unilaterally deafened CI users. Further, we particularly characterised the contribution of CI usage in binaural integration by calculating the CI-related binaural benefit. The capability of binaural integration at the brainstem and higher-order cortical levels would be reflected by an improvement in speech recognition under dichotic listening compared to a monotic listening condition. Additionally, a substantial CI-related contribution is expected for low segmentation rates, while minimal or no contribution is anticipated for high rates.

In contrast to previous studies on binaural integration, where the focus has been on the assessment of processes at the level of the auditory brainstem, the present study investigates the binaural

T. Wesarg and N. Richter contributed equally to this work.

\section{KARGER 125\%}

E-Mail karger@karger.com www.karger.com/aud
(C) 2015 S. Karger AG, Base 1420-3030/15/0207-0073\$39.50/0

Karger $\checkmark$ pen access

This is an Open Access article licensed under the terms of the Creative Commons Attribution-NonCommercial 3.0 Unported license (CC BY-NC) (www.karger.com/OA-license), applicable to the online version of the article only. Distribution permitted for non-commercial purposes only. 
Table 1. Characteristics of the patients with severe to profound UHL

\begin{tabular}{|c|c|c|c|c|c|c|c|c|c|c|}
\hline \multirow{2}{*}{$\begin{array}{l}\text { Subject } \\
\text { No. }\end{array}$} & \multirow{2}{*}{ Gender } & \multirow{2}{*}{$\begin{array}{l}\text { Age, } \\
\text { years }\end{array}$} & \multirow[t]{2}{*}{ Aetiology } & \multirow{2}{*}{$\begin{array}{l}\text { Duration } \\
\text { of severe to } \\
\text { profound } \\
\text { UHL, year }\end{array}$} & \multirow{2}{*}{$\begin{array}{l}\text { CI } \\
\text { ear }\end{array}$} & \multicolumn{2}{|c|}{ PTA, dB HL } & \multirow[t]{2}{*}{ CI type } & \multirow{2}{*}{$\begin{array}{l}\text { CI speech } \\
\text { processor }\end{array}$} & \multirow{2}{*}{$\begin{array}{l}\text { CI use, } \\
\text { years }\end{array}$} \\
\hline & & & & & & CI ear & $\begin{array}{l}\text { contra- } \\
\text { lateral ear }\end{array}$ & & & \\
\hline 1 & M & 47.7 & Acoustic trauma & 1.0 & $\mathrm{R}$ & $>120$ & 11 & Nucleus CI512 & CP810 & 3.5 \\
\hline 2 & $\mathrm{~F}$ & 45.6 & Labyrinthitis & 0.8 & $\mathrm{~L}$ & $>120$ & 8 & Nucleus CI24RE (CA) & Freedom SP & 4.5 \\
\hline 3 & $\mathrm{~F}$ & 31.0 & Cogan I syndrome & 0.8 & $\mathrm{R}$ & $>120$ & 7 & Nucleus CI512 & CP810 & 2.2 \\
\hline 4 & M & 35.7 & Ear surgery & 0.3 & $\mathrm{R}$ & 117 & 12 & Nucleus CI24RE (CA) & Freedom SP & 4.2 \\
\hline 5 & $\mathrm{~F}$ & 52.8 & Acute hearing loss & 3.5 & $\mathrm{~L}$ & $>120$ & 4 & Nucleus CI24RE (CA) & Freedom SP & 5.2 \\
\hline 6 & M & 54.6 & Acute hearing loss & 1.6 & $\mathrm{~L}$ & $>120$ & 20 & Nucleus CI512 & CP810 & 3.3 \\
\hline 7 & $\mathrm{M}$ & 59.4 & Acute hearing loss & 0.8 & $\mathrm{~L}$ & $>120$ & 8 & Nucleus CI24RE (CA) & Freedom SP & 4.5 \\
\hline 8 & $\mathrm{~F}$ & 42.6 & Acute hearing loss & 3.1 & $\mathrm{~L}$ & $>120$ & 11 & Nucleus CI24RE (CA) & CP810 & 4.9 \\
\hline 9 & $\mathrm{~F}$ & 51.3 & Morbus Ménière & 13.4 & $\mathrm{~L}$ & $>120$ & 4 & Nucleus CI24RE (CA) & Freedom SP & 4.6 \\
\hline 10 & M & 45.1 & Meningitis & 0.1 & $\mathrm{R}$ & $>120$ & 11 & Nucleus CI24RE (CA) & CP810 & 0.6 \\
\hline
\end{tabular}

integration and binaural benefit obtained from CI usage based on processing mechanisms associated with brainstem and cortical structures. Furthermore, interpretation of the findings from the present study is considered to add to the knowledge on binaural integration processes, which are expanded up to neurophysiological correlates at the cortical level beyond the auditory brainstem.

\section{Materials and Methods}

Participants. A total of 10 adult CI subjects ( 5 females and 5 males; age range: $31.0-59.4$ years, mean age: 46.6 years) with acquired, sensorineural, severe to profound UHL were enrolled in the study. At inclusion, all of them showed a profound hearing loss [air conduction pure-tone average (PTA) $>110 \mathrm{~dB} \mathrm{HL}$ ] in the implanted ear and NH thresholds (air conduction PTA $\leq 20 \mathrm{~dB} \mathrm{HL}$ ) in the contralateral ear, and had at least 2 years of CI experience except for subject S10 (7 months). With their CI, the group obtained an average monosyllabic word score of $60.5 \%$ (range 50 $75 \%)$ at $65 \mathrm{~dB}$ SPL. Pertinent individual subject characteristics are shown in table 1 . This study was designed and conducted in accordance with the Declaration of Helsinki and approved by the Ethics Committee of the University of Freiburg. All subjects provided written consent.

Alternating Speech Perception Test. To test for binaural integration, a modified version of the RASP test according to Calearo [1957] and Bocca and Calearo [1963] was employed. In this test, sentences from the German OlKiSa (Oldenburg Sentence Test for Children) [Neumann et al., 2012], e.g. 'Drei grosse Blumen', are applied as speech stimuli, and speech recognition is assessed as percentage of correctly recognised words. For each subject, speech recognition was measured using one list of 14 sentences in each of three listening conditions: monotic with the CI ear; monotic with the contralateral $\mathrm{NH}$ ear, and dichotic. The order of test conditions was randomised across subjects. Before administering the speech recognition tests, all subjects underwent extensive training for familiarisation to the test procedure and material.

The speech stimuli were split into sentence segments of equal duration, which were grouped into sentence intervals, each consisting of four segments (fig. 1a). In case of monotic stimulation of the $\mathrm{NH}$ ear, the first segment of each interval was presented (fig. 1b), while monotic stimulation of the CI ear comprised the presentation of the second and third segment of each interval, i.e.

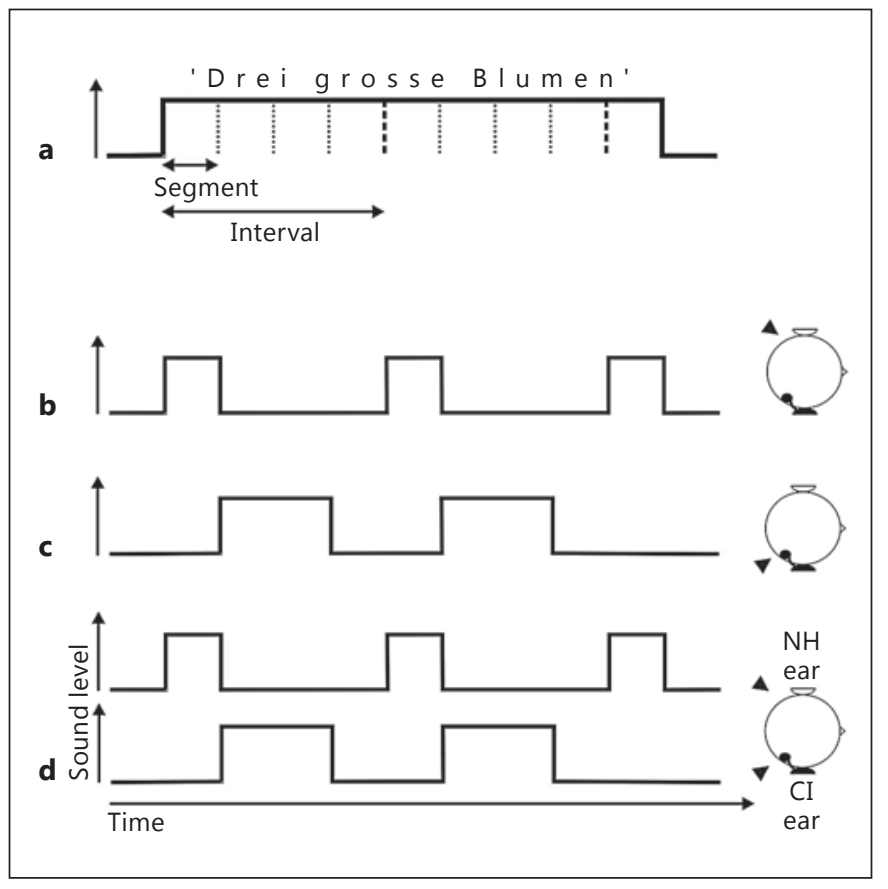

Fig. 1. Stimulation paradigm. a Illustration of the splitting of an exemplary OlKiSa sentence 'Drei grosse Blumen' (solid line) into sentence segments (dotted lines). b-d Distinct listening arrangements with monotic presentation of stimulus segments to the $\mathrm{NH}$ ear (b), of 'double segments' to the CI ear (c) and with dichotic stimulus presentation of side-alternating sentence segments to both the $\mathrm{NH}$ and the $\mathrm{CI}$ ear $(\mathbf{d})$. The segmentation rate is $1 / \mathrm{seg}$ ment duration, and the ratio of stimulus segments transmitted to the $\mathrm{NH}$ and $\mathrm{CI}$ ear is $1-2$.

a double segment (fig. 1c). Figure 1d shows the dichotic stimulation scheme with alternating presentation of the first interval segments to the $\mathrm{NH}$ ear and the following interval double segments to the CI ear without presentation of the fourth interval segments.

Speech recognition for the monotic NH ear, monotic CI ear and dichotic listening conditions was evaluated for five segment 


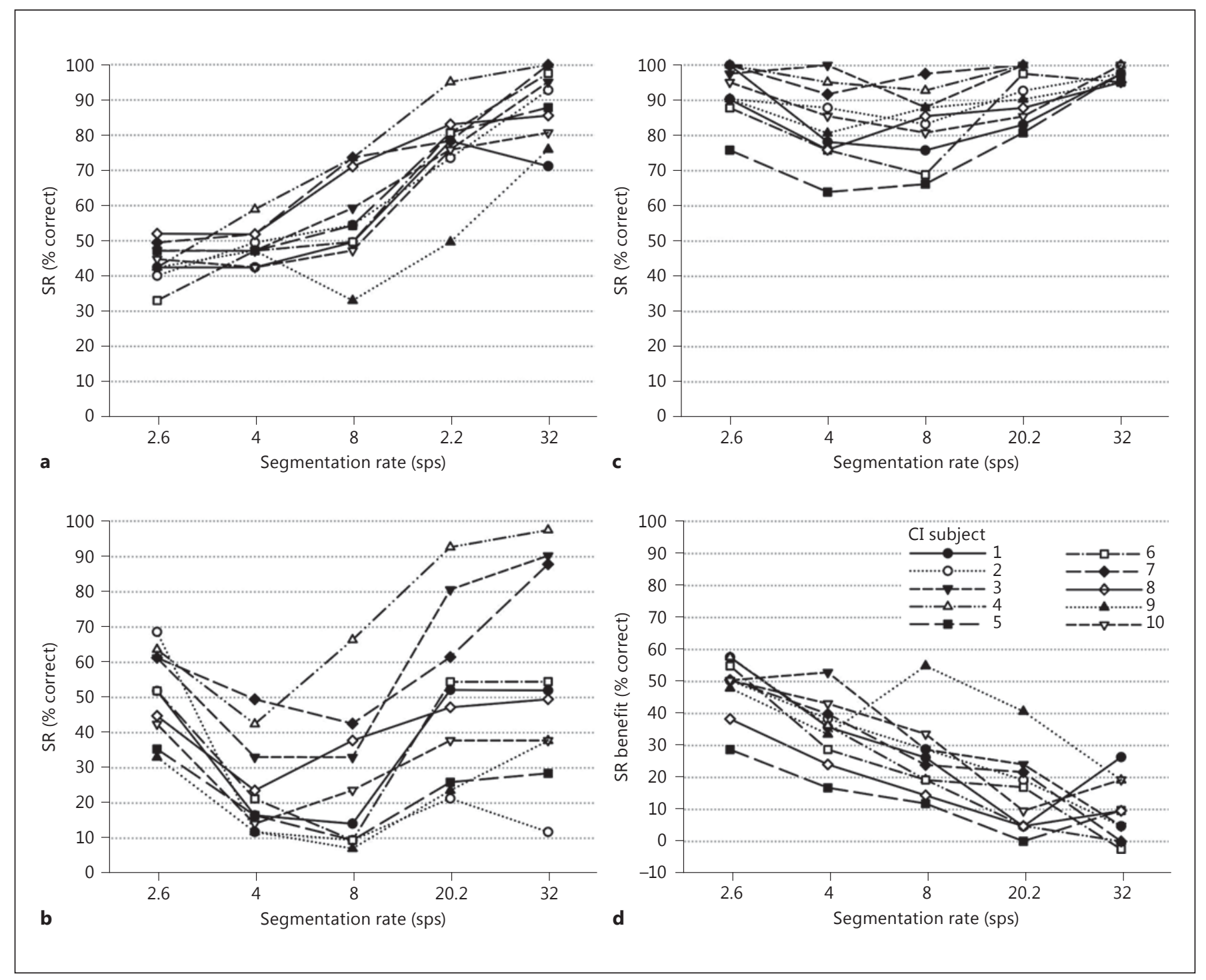

Fig. 2. Speech recognition (SR) in CI users with UHL $(n=10)$ for monotic stimulus presentation to the $\mathrm{NH}$ ear (a), to the $\mathrm{CI}$ ear (b) and for dichotic stimulus presentation (c). $\mathbf{d}$ The binaural benefit by CI usage was calculated as the difference between speech recog-

durations (31.2, 49.5, 125, 250 and $384.6 \mathrm{~ms})$ corresponding to segmentation rates of $32,20.2,8,4$ and 2.6 sps, respectively. Different segmentation rates were applied to assess the effect of segment duration on monotic speech recognition, which is reported to be maximum at high segmentation rates of 20 sps and minimum at low rates of 4 sps or less in normal hearing subjects [Lehnhardt, 2001]. Similarly, different segmentation rates were applied to assess dichotic speech recognition as well as binaural benefit per test condition and material used. The relationship between the number of segments per interval presented to the $\mathrm{CI}$ and $\mathrm{NH}$ ear, respectively, and thus between applied stimulus durations in a 2:1 ratio, was chosen in order to limit the occurrence of floor effects for speech recognition with the $\mathrm{CI}$ ear as well as ceiling effects for nition under dichotic and monotic $\mathrm{NH}$ ear listening conditions. Speech recognition values are expressed as percent correct answers and are shown for five segmentation rates tested.

speech recognition with the $\mathrm{NH}$ ear. Different segmentation rates were applied to identify the most suitable segmentation rate to ensure accurate measure of performance in monotic stimulation conditions and, thus, adequate sensitivity to the binaural benefit in the dichotic stimulation condition.

Speech stimuli were presented to the $\mathrm{NH}$ ear via headphone (Sennheiser HDA 200) and to the CI ear via an audio cable attached to the audio input socket of the CI speech processor. The presentation level before sentence splitting was $65 \mathrm{~dB}$ SPL for NH ear stimulation. For CI ear stimulation, the root mean square voltage of the electrical speech stimuli was chosen to elicit the same loudness as the corresponding acoustic speech stimuli with $65 \mathrm{~dB}$ SPL presented to the microphone of the speech processors. Stimu- 
Table 2. Results of CI recipients with UHL analysed by repeated-measure ANOVA and all pairwise multiple comparison procedures on speech recognition as correct responses for the monotic stimulation condition (a), including the factors ear (two levels: $\mathrm{NH}$ ear and $\mathrm{CI}$ ear) and segmentation rate (five levels: 2.6, 4, 8, 20.2 and 32 sps) and for dichotic versus monotic listening condition (b), including the factors listening condition (two levels: monotic and dichotic) and segmentation rate, and results of one-way ANOVA for binaural benefit (c), as well as results of pairwise multiple comparisons (Bonferroni's $\mathrm{t}$ test; $\mathbf{d}$ )

\begin{tabular}{lll}
\hline Speech recognition in unilateral CI recipients & Correct responses, \% & p value \\
\hline $\begin{array}{l}\text { a Monotic listening conditions } \\
\begin{array}{l}\text { Repeated-measure ANOVA with factors } \\
\text { Ear }\end{array}\end{array}$ & & \\
$\quad \begin{array}{l}\text { Segmentation rate } \\
\quad \text { Interaction } \\
\quad \text { Ear } \times \text { segmentation rate }\end{array}$ & $\mathrm{F}(1,9)=28.7$ & $<0.001$ \\
& $\mathrm{~F}(4,36)=38.9$ & $<0.001$ \\
$\begin{array}{l}\text { b Dichotic versus monotic listening condition } \\
\text { Repeated-measure ANOVA with factors } \\
\quad \text { Listening condition } \\
\quad \text { Segmentation rate }\end{array}$ & $<0.001$ \\
\hline $\begin{array}{l}\text { c Binaural benefit } \\
\text { One-way ANOVA }\end{array}$ & $\mathrm{F}(1,9)=200.7$ & \\
\hline
\end{tabular}

d Bonferroni's correction for multiple comparisons

\begin{tabular}{llll}
\hline Comparison & Difference in means & $\mathrm{t}$ & p value \\
\hline 2.6 vs. 32 & 39.5 & 8.24 & $<0.001$ \\
2.6 vs. 20.2 & 33.9 & 7.09 & $<0.001$ \\
2.6 vs. 8 & 22.5 & 4.705 & $<0.001$ \\
2.6 vs. 4 & 13.7 & 2.862 & 0.064 \\
4 vs. 32 & 25.7 & 5.378 & $<0.001$ \\
4 vs. 20.2 & 20.2 & 4.228 & $<0.001$ \\
4 vs. 8 & 8.8 & 1.842 & 0.72 \\
8 vs. 32 & 16.9 & 3.536 & 0.01 \\
8 vs. 20.2 & 11.4 & 2.385 & 0.213 \\
20.2 vs. 32 & 5.5 & 1.15 & 1 \\
\hline
\end{tabular}

Significant $\mathrm{p}$ and $\mathrm{t}$ values are italicised. lus presentation as well as speech test flow control were managed with a desktop PC with a RME Fireface UC (Audio AG, Haimhausen, Germany) sound card and a software running in Matlab R2011b (MathWorks Inc., Natick, Mass., USA).

Data Analysis. For each subject and segmentation rate, the binaural benefit of using the $\mathrm{CI}$ for dichotic speech recognition, i.e. its contribution to binaural integration, is determined as the difference between dichotic speech recognition and monotic speech recognition using the $\mathrm{NH}$ ear only.

Differences in monotic speech recognitions were analysed by means of a repeated-measure ANOVA including the factors 'ear' (two levels: NH ear and CI ear) and 'segmentation rate' (five levels: $2.6,4,8,20.2$ and $32 \mathrm{sps}$ ). To analyse speech recognition under dichotic and monotic listening conditions, results obtained for the $\mathrm{NH}$ ear and the respective $\mathrm{CI}$ ear were collapsed and a repeatedmeasure ANOVA including the factors 'listening condition' (two levels: monotic and dichotic) and 'segmentation rate' was performed.

The respective binaural benefit for different segmentation rates was tested against zero level with one-sample, two-tailed Student's $\mathrm{t}$ tests. In addition, a one-way ANOVA with factor segmentation rate was conducted. Greenhouse-Geisser-corrected degrees of freedom were applied whenever appropriate and Bonferroni's correction for $\mathrm{t}$ tests were used for pairwise multiple comparisons.

\section{Results}

Individual data of monotic speech recognition with the contralateral $\mathrm{NH}$ ear are plotted in figure 2a. Speech recognition shows rather small interindividual variability at the two lowest segmentation rates and increases with growing segmentation rate for almost all subjects, and the group medians ranged from $43.6 \%$ at 2.6 sps to $90.3 \%$ at 32 sps. Figure $2 \mathrm{~b}$ depicts individual monotic CI ear speech recognition data showing large interindividual differences particularly at medium and higher segmentation rates. For the majority of subjects, speech recognition is smallest at segmentation rates of 4 and 8 sps, which also holds for the group median (18.9\% each). At the lowest and two highest segmentation rates of 2.6, 20.2 and $32 \mathrm{sps}$, the respective speech recognition group median was much larger (51.9, 49.7 and 50.8\%, respectively). At the lowest segmentation rate of $2.6 \mathrm{sps}$, the group median monotic speech recognition with the CI ear is somewhat larger than with the $\mathrm{NH}$ ear (51.9 vs. $43.6 \%$ ), while at all other segmentation rates, the monotic $\mathrm{NH}$ ear speech recognition group median is superior (table 2a, significant main effect of factor ear, significant interaction of factor ear and factor segmentation rate).

Under dichotic presentation, individual speech recognition exceeds $60 \%$ in all subjects and at all segmentation rates. It seems to be widely independent of segmentation rate in the majority of sub- 
jects and contains rather small interindividual variability particularly at the two highest segmentation rates (fig. 1c). Group median speech recognition varies only from $83.1 \%$ at 4 sps to $97.6 \%$ at 32 sps, showing a ceiling effect at the highest rate (table $2 \mathrm{~b}$, significant effect of factor segmentation rate). Figure $1 \mathrm{~d}$ shows the individual data of the binaural benefit of CI usage for dichotic speech recognition, which have medium-size interindividual differences (onesample, two-tailed Student's t test: all p < 0.05). Six of the 10 subjects demonstrated a binaural benefit at all segmentation rates. At the lower and medium segmentation rates from 2.6 to $8 \mathrm{sps}$, all subjects showed a binaural benefit in the range from 11.8 to $57.6 \%$, while the majority of subjects also had a binaural benefit at the higher rates of 20.2 and 32 sps. Based on the group median, a binaural benefit was noted at all segmentation rates and decreased with increasing segmentation rate from $50.4 \%$ at 2.6 sps to $7.2 \%$ at 32 sps (table 2c, d).

\section{Discussion}

The present study investigated binaural integration in the central auditory system of CI recipients with acquired, profound UHL and $\mathrm{NH}$ in the contralateral ear. Applying a modified version of the RASP test, speech recognition under monotic and dichotic listening arrangements was determined, and binaural benefit by CI was assessed. Whereas the interpretation of findings of previous studies is limited to processing mechanisms at the level of the auditory brainstem, the present study expanded the existing knowledge on binaural integration up to neurophysiological correlates that accompany the processing mechanisms associated with structures at the brainstem and cortical levels.

Results obtained under monotic listening conditions show that CI recipients are generally able to understand speech by processing sentence segments presented with a quick rate to either the $\mathrm{NH}$ or $\mathrm{CI}$ ear. Respective speech recognition of the CI ear is poorer compared to the $\mathrm{NH}$ ear, which is in accordance with previous reports on CI recipients [Zeng, 2004; Jin et al., 2014]. Interestingly, the monotic speech recognition is differently influenced by the segmentation rate in the $\mathrm{NH}$ and $\mathrm{CI}$ ears listening conditions, respectively. For the NH ear listening, speech recognition gradually improves as a function of increasing segmentation rate. For the CI ear listening, generally also highest recognition values were found for high segmentation rates, but lowest speech recognition was not noted at lowest but at medium segmentation rates. This discrepancy between results for the $\mathrm{NH}$ and $\mathrm{CI}$ ear might be explained by differences in segments between the $\mathrm{NH}$ and $\mathrm{CI}$ ears and in sentence segment stimuli presented between the listening conditions. However, the present findings demonstrating small intersubject variability and reduced binaural benefit at high segmentation rates in the dichotic condition are putatively based on the ceiling effects obtained under monotic NH ear conditions and suggest an insensitivity of the present RASP test at high segmentation rates. In this respect, the present results indicate that the segmentation rate of 2.6 sps is the most suitable to guarantee comparable performance rates between $\mathrm{CI}$ and $\mathrm{NH}$ ear conditions, as it will exclude monaural floor and ceiling effects, which are needed in order to adequately assess binaural integration as a method to reflect the binaural benefit in CI recipients.

The ability of binaural integration was characterised by grading speech recognition in case of rapidly alternating presentation of speech segments to the $\mathrm{NH}$ and $\mathrm{CI}$ ears, respectively. The present results - particularly for low and medium segmentation rates show that speech recognition was generally improved under dichotic compared to monotic listening conditions. From these results, it can be concluded that bilaterally electrically and acoustically transmitted information can be integrated binaurally in the central auditory system, obviously at the level of the brainstem and the cortex [Medwetsky, 2002; Stach, 2007].

The present finding of a binaural benefit in CI recipients, which was demonstrated by superior speech recognition for the dichotic listening mode compared to the monotic CI ear mode, indicates that the central auditory system at the brainstem and cortical level is able to integrate information derived from bimodal input consisting of acoustic stimuli via the $\mathrm{NH}$ ear and electric stimuli via the CI ear to auditory units. CI users having difficulties in performing the present RASP test may still be able to demonstrate a binaural benefit via measures of the localisation test, which is currently the preferred test to clinically assess binaural integration at the level of the brainstem. However, the present RASP test applied at a low segmentation rate is suitable as an additive test to investigate the binaural integration based on processing mechanisms associated with structures of the ascending auditory system at the cortical level.

\section{Conclusion}

The present study showed that under monotic listening conditions, CI users with UHL are generally able to process sentence segments presented to either the $\mathrm{NH}$ or the CI ear, with the respective speech recognition performance being poorer for the CI ear compared to the $\mathrm{NH}$ ear. Under dichotic listening conditions, speech recognition was considerably improved, which hints to the ability to integrate bilaterally presented, electrically and acoustically transmitted information via binaural processing in the central auditory system. Finally, it can be suggested that while CI recipients with diffuse cortical problems might exhibit binaural benefits in localisation tests, they may nonetheless demonstrate a smaller or negligible binaural benefit in the present RASP test, which inherently includes processing at the cortical level.

\section{Acknowledgement}

We would like to express our appreciation to all CI users for their willingness and participation in our experimental sessions. We would also like to thank Konstantin Wiebe for his technical support when developing the experimental set-up. We thank Josie Wyss and two anonymous reviewers for important suggestions on previous drafts of this paper. The authors also acknowledge the association 'Taube Kinder lernen Hören e.V.', which considerably supports the CI rehabilitation centre and the hearing research on cochlear implant listeners in Freiburg.

\section{Disclosure Statement}

Horst Hessel $(\mathrm{PhD})$ is an employee of Cochlear Deutschland $\mathrm{GmbH} \& \mathrm{Co}$. KG. This research was partly funded by Cochlear Deutschland GmbH \& Co. KG. 


\section{References}

Arndt S, Aschendorff A, Laszig R, Beck R, Schild C, Kroeger S, Ihorst G, Wesarg T: Comparison of pseudobinaural hearing to real binaural hearing rehabilitation after cochlear implantation in patients with unilateral deafness and tinnitus. Otol Neurotol 2011;32:39-47.

Bocca E, Calearo C: Central hearing processes; in Jerger J (ed): Modern Developments in Audiology, ed 1. New York, Academic Press, 1963, pp 337-370.

Buechner A, Brendel M, Lesinski-Schiedat A, Wenzel G, Frohne-Buechner $\mathrm{C}$, Jaeger B, Lenarz T: Cochlear implantation in unilateral deaf subjects associated with ipsilateral tinnitus. Otol Neurotol 2010;31:1381-1385.

Calearo C: Detection of malingering by periodically switched speech. Laryngoscope 1957;67:131-136.

-Jacob R, Stelzig Y, Nopp P, Schleich P: Audiological results with cochlear implants for single-sided deafness (in German). HNO 2011;59:453-460.

-Jin SH, Liu C, Sladen DP: The effects of aging on speech perception in noise: comparison between normal-hearing and cochlear-implant listeners. J Am Acad Audiol 2014;25:656-665.

Laszig R, Arndt S, Aschendorff A, Wesarg T: Is a cochlear implant the only option to restore binaural hearing? Cochlear Science and Research Seminar Asymmetric Hearing Loss, Toulouse, 2014, unpubl results.

Lehnhardt E: Zentrale Hördiagnostik; in Lehnhardt E, Laszig R (eds): Praxis der Audiometrie, ed 8. Stuttgart, Thieme, 2001, pp 227-237.
Medwetsky L: Central auditory processing; in Katz J (ed): Handbook of Clinical Audiology, ed 8. Baltimore, Lippincott Williams \& Wilkins, 2002, pp 495-524.

Neumann K, Baumeister N, Baumann U, Sick U, Euler HA, Weissgerber T: Speech audiometry in quiet with the Oldenburg Sentence Test for Children. Int J Audiol 2012;51:157-163.

Stach BA: Diagnosing auditory processing disorders in adults; in Roeser RJ, Valente M, Hosford-Dunn H (eds): Audiology. Diagnosis, ed 2. New York, Thieme, 2007, pp 356-379.

-Vermeire K, Van de Heyning P: Binaural hearing after cochlear implantation in subjects with unilateral sensorineural deafness and tinnitus. Audiol Neurootol 2009;14:163-171.

Zeng FG: Trends in cochlear implants. Trends Amplif 2004;8:1-34.

Correspondending author:

Dr. Thomas Wesarg

Department of Otorhinolaryngology, Head and Neck Surgery

University Medical Centre Freiburg

Killianstrasse 5, DE-79106 Freiburg (Germany)

E-Mail thomas.wesarg@ uniklinik-freiburg.de 